\title{
Photographies de Samori Touré : de la carte postale coloniale aux pochettes de vinyles
}

Le devenir d'une icône

Photographs of Samori Touré: From the Colonial Postcard to Record Covers.

Circulations of an Iconic Picture

\section{Elara Bertho}

\section{CpenEdition}

Journals

Édition électronique

URL : https://journals.openedition.org/etudesafricaines/22087

DOI : 10.4000 /etudesafricaines.22087

ISSN : $1777-5353$

Éditeur

Éditions de l'EHESS

\section{Édition imprimée}

Date de publication : 1 juin 2018

Pagination : 301-322

ISBN : 978-2-7132-2742-4

ISSN : 0008-0055

\section{Référence électronique}

Elara Bertho, «Photographies de Samori Touré : de la carte postale coloniale aux pochettes de vinyles », Cahiers d'études africaines [En ligne], 230 | 2018, mis en ligne le 01 juin 2020, consulté le 21 septembre 2021. URL : http://journals.openedition.org/etudesafricaines/22087 ; DOI : https://doi.org/ 10.4000/etudesafricaines.22087 


\title{
Elara Bertho
}

\section{Photographies de Samori Touré: de la carte postale coloniale aux pochettes de vinyles}

\author{
Le devenir d'une icône
}

Paradoxalement, l'image contestataire n'est pas nécessairement l'image d'une contestation ${ }^{1}$. Plus exactement, l'image lue comme contestataire n'est pas forcément l'image d'une manifestation ou d'un soulèvement. Dans l'exposition qu'il consacre aux « Soulèvements », Georges Didi-Huberman choisit comme "geste» iconique de la contestation le bras d'un manifestant qui vient tout juste de lancer un pavé contre la police, saisi sur le vif par le photographe Gilles Caron ${ }^{2}$ en 1969, au cours d'une manifestation anticatholique à Londonderry (Didi-Huberman et al. 2016 : 138).

Or, si le geste est certes saisissant, ce n'est pas l'unique ressort de la portée contestataire d'une image : en effet, les usages et les circulations subversives d'une photographie peuvent au contraire se nourrir du grand calme qui s'en dégage, aussi paradoxal que cela puisse paraître de prime abord. C'est précisément à une étude du calme que nous voudrions nous livrer ici, en pointant à quel point il dissimule des rapports de forces induits entre le sujet et l'objet de la photographie. Surtout, à un autre niveau, c'est la vie de la photographie détachée de son contexte de production qui peut porter la contestation : ce sont alors les usages contestataires d'un cliché en apparence particulièrement serein, mais qui a connu de formidables et nombreuses réutilisations, vives et subversives, qui permettent d'en comprendre la portée et l'aura.

1. Une bourse de la British Academy (2016-2017) a servi à financer les recherches menées en Guinée en collaboration avec Marie Rodet (Senior Lecturer, SOAS University). Je remercie les relecteurs externes qui m'ont apporté de précieuses informations ainsi que Daniela Moreau.

2. G. Caron, Manifestations anticatholiques à Londonderry, Genève, Fondation Gilles Caron, 1969. 
Lorsqu'en 1898, Henri Gaden et Henri Gouraud photographient leur célèbre prisonnier, Samori Touré, qui a tenu tête aux troupes coloniales françaises et britanniques pendant plus de vingt ans, ils immortalisent avant tout la victoire de l'armée française et l'avancée de la conquête coloniale. Très admiratif de leur détenu (Dilley 2015), ils en dressent un portrait où il est présenté assis, tenant un Coran entre les mains, fixant résolument le spectateur. Les officiers français sont loin d'imaginer à quel point cette photographie sera réutilisée, notamment pour magnifier la lutte anticoloniale et servir la contestation de l'hégémonie française en Afrique de l'Ouest.

À partir d'un important travail d'archives ${ }^{3}$, nous proposons de retracer les parcours et usages des différentes photographies de Samori Touré lors de sa capture, en croisant les différents acteurs qui l'ont accompagné : Henri Gaden, Henri Gouraud, ainsi que le capitaine Tamburini, plus méconnu, dont certains des clichés inédits ont récemment été retrouvés par nous au Musée de l'Armée 4 . Plus particulièrement, le fameux portrait au Coran, parmi ces différentes séries de clichés de 1898 et 1899, a connu un vif succès populaire dès l'époque coloniale, via des cartes postales ou des reproductions en illustrés ou dans des magazines. À l'indépendance de la Guinée, Sékou Touré, qui se revendique d'une filiation symbolique et biologique ${ }^{5}$ de Samori Touré (Goerg 2011), fait un usage extrêmement important de ce portrait, tout en inversant la lecture : ce n'est plus une prise de guerre mais la célébration d'un père de la nouvelle patrie, mort en martyr et en exil en 1900, dont il s'agit de magnifier la mémoire (Pauthier 2012, 2014 ; Bertho 2015). Le Bembeya Jazz National réutilise le cliché, en le colorisant de multiples manières, pour dresser un éloge du nouveau régime socialiste, via la reprise de la photographie coloniale ${ }^{6}$. La statuaire contemporaine sur Samori à Conakry et dans le nord de la Guinée illustre l'impact de ce cliché dans la formation d'une « imagerie » populaire socialiste.

Cette étude entend dresser une archéologie de la fabrique d'une imagerie héroïque : en se fondant sur une pratique coloniale (la célébration de la capture d'un ennemi sur le modèle des tableaux de chasse, des «massacres »), les Indépendances viennent opérer une inversion du regard sur ces clichés, portant désormais la contestation tout à la fois anticoloniale et anticapitaliste que

3. Archives nationales d'Outre-mer (ANOM), Aix-en-Provence. Service historique de la Défense (SHD), Fort de Vincennes. Musée de l'Armée, Hôtel des Invalides.

4. Sur la capture de Samori Touré, voir D’Andurain (2012) et Person (1968-1975).

5. Sékou Touré se dit descendant de Bagbè Ramata Touré, l'une des filles de Samori Touré.

6. Notons que d'autres cas de répétition à l'identique sont construits sur le même modèle : ainsi de celui d'Ahmadou Bamba, où l'unique photographie qui le représente est dupliquée à l'envi au Sénégal et plus largement en Afrique de l'Ouest. Voir l'article de G. Paoletti dans ce même numéro. 
Sékou Touré $(1969,1972)$ menait par ailleurs dans ses œuvres littéraires et ses discours.

Comment l'image porte-t-elle la contestation ? Quelles lectures contestataires peuvent en être faites et à quelles conditions? Comment les usages contestataires de l'image circulent-ils ? Que dit le calme d'une photographie ? Quels usages sont faits d'un regard? Ce sont ces questions sur le « devenir iconique » du portrait de Samori Touré que nous interrogeons ici. Reprenant la notion de punctum au célèbre essai La chambre claire de Roland Barthes (1980), Jacques Rancière (2013 : 19-23) analyse « ce qui nous frappe » dans une photographie, autrement dit, cette puissance effective du sentiment de présence charnelle de l'objet que nous éprouvons à la vue d'une photographie. Il en déduit deux types de puissance de l'image : «l'image comme présence sensible brute et l'image comme discours chiffrant une histoire. » Dans le mutisme du sourire du sujet de la photographie se lit effectivement un indéniable sentiment de présence du corps : c'est le premier niveau de lecture, où la photographie est un document historique, attestant de la rencontre entre un prisonnier de guerre et des officiers français (devenus également ses photographes) dans un rapport de force ambigu entre ces hommes. Deuxième niveau de lecture : le sourire, ce punctum, contient le chiffre d'une histoire, qu'il reste à établir et à interpréter. Il y a une «mise en scène discursive » (Rancière $2013: 38$ ) à retracer, derrière le silence de ce cliché : ce que le cliché ne dit pas, ce que la quiétude de la pose prend soin de ne pas dire. C'est cette double lecture de l'aura de l'image qu'il s'agit d'effectuer pour, si ce n'est lire «à rebrousse poil» les sources (Benjamin [1940] 2000: 433), du moins lire « against the camera » (Tupitsyn 2006: 103), afin de rendre au calme de ce cliché toutes ses portées contestataires.

\section{Samori Touré à sa capture: I'empereur et le photographe}

Une capture contestée: une guerre des images

Le 29 septembre 1898, le capitaine Gouraud arrête Samori Touré et obtient la reddition des quelques 100000 personnes qui l'accompagnaient dans sa fuite (d'Andurain 2012 : 32-44). Cette guerre menée pendant plus de vingt ans contre les troupes coloniales, tant françaises que britanniques, entrecoupée de traités de paix et de redéfinitions successives de frontières, s'achève donc sur cette prise très célèbre : celui que les officiers appelaient le «Napoléon des savanes » s'est rendu. Abondamment documentée, cette guerre de positions a fait l'objet de bien des fantasmes et, dès sa capture, de multiples images, 
gravures, photographies parviennent à Paris : parfois contradictoires, parfois mensongères, elles révèlent toutes le grand intérêt que portait le public à cette figure de chef africain. Ce qui est d'emblée frappant, c'est en effet la grande profusion iconographique concernant Samori Touré et ce, dès l'annonce de sa capture. Les clichés ne parvenant qu'avec le retour des troupes en métropole, c'est d'abord par les gravures que la nouvelle se répand.

Fait significatif, Le supplément illustré du Petit Journal affiche en pleine page une gravure tout à fait inventée et fantaisiste de la capture (fig. 1) : elle présente le lieutenant Jacquin sur un cheval noir lancé au galop, le sabre au clair, attrapant Samori Touré au col de son large manteau blanc, forçant son cheval, blanc également, à ralentir sa course. Hérö̈que, cette scène aux colorations épiques a tout d'une image d'Épinal de la conquête coloniale : deux hommes s'affrontent au premier plan — un Blanc, un Noir — dans une parfaite symétrie des corps et des lignes. Au fond, la charge d'un cavalier, le sabre au clair également, complète une vision plus indistincte des troupes auxiliaires. Ce qui frappe surtout le spectateur, c'est l'expression d'étonnement de Samori Touré, pris en pleine cavale, littéralement, désarmé, déjà vaincu.

Or, cette scène est une reconstitution tout à fait mensongère de l'épisode, destinée à glorifier le lieutenant Jacquin qui, d'ailleurs, n'a pas dirigé l'opération de capture de Samori. Cette gravure est en cela parfaitement significative de la «guerre au loin » que Sylvain Venayre (2016) a étudiée au sujet des reportages de Pierre Loti pour Le Figaro, dans l'Annam (voir également Bancel, Blanchard \& Gervereau 1993). Les photographes de presse et la figure du journaliste de terrain n'existaient pas encore et les reconstitutions graphiques obéissaient donc aux enjeux de pouvoir du moment. Ainsi, la paternité de la capture de Samori Touré a fait l'objet de nombreuses contestations dans les rangs de l'armée française. Julie d'Andurain (2012 : 46) décrit comment le capitaine Gouraud et le chef de bataillon Lartigue se sont disputé la gloire de ce haut fait militaire auprès de leurs supérieurs, par lettres interposées, suscitant une grande correspondance. Cette rivalité s'exprime également par voie de presse, et c'est le lieutenant Jacquin qui semble gagner, dans un premier temps, la bataille médiatique avec cette «Une » fracassante autant qu'inventée. Gouraud et Lartigue s'empresseront de reprendre cette guerre des images en répondant par d'autres images, plus conformes au déroulement des opérations. Ainsi le «journal de marche » de Gouraud (1899 : 7-13) est-il diffusé par plusieurs canaux, notamment dans le célèbre Bulletin du Comité de l'Afrique française. Plus intéressante car illustrée est la version parue dans Lectures pour tous. Revue Universelle illustrée (Gouraud 1899b : 961-976) « avec treize illustrations et deux cartes », « dessins de Mme Crampel d'après les indications de l'auteur », où figure en première 
page Samori Touré fuyant à pied, poursuivi par Bratières et, derrière, par une masse indistincte de tirailleurs.

L'arrestation de Samori Touré a eu lieu suite à une offensive de Lartigue, qui dépêche en reconnaissance le capitaine Gouraud (Gouraud 1939; Person 1975, III : 2011-2014) le 20 septembre 1898. Huit jours plus tard, à Guélémou, Henri Gouraud est à la lisière du camp de Samori Touré. Le 29 au lever du jour, il ordonne à la colonne de traverser le camp sans tirer de coup de feu et de se saisir de 1'Almami ${ }^{7}$. Jacquin est dépêché en avant, et ce sont deux tirailleurs, Bandya Tunkara et Filifin Keita qui s'emparent de Samori, occupé à lire matinalement le Coran à l'entrée de sa case. S'enfuyant, il est cerné par les deux hommes et par le sergent Bratières. Quelques remarques sur ce rapide résumé des faits : Lartigue, qui dirigeait les opérations, n'était pas sur place et était furieux d'avoir été dépassé par Gouraud. Ce dernier a mené une opération tactique remarquable, sans effusion de sang aucune. Enfin, ce sont des tirailleurs qui ont, formellement, arrêté Samori. Bien évidemment, la Une du Petit Journal illustré a opéré une sélection stratégique des informations afin de magnifier l'épisode.

Ce «faux » iconographique produit par le journal a ceci d'intéressant qu'il révèle non seulement toutes les dissensions internes à l'armée coloniale, mais aussi ce qu'il était visuellement acceptable ou non, de diffuser à la fin du XIX ${ }^{\mathrm{e}}$ siècle. Une arrestation en courant, à pied, d'un chef noir par deux tirailleurs noirs avait moins de panache qu'une charge au galop menée par un officier blanc en uniforme, galons au vent et sabre au clair. En sous-texte également : cet indicible que la guerre coloniale ne pouvait se faire que par des Blancs, avec tous les impensés racistes que cela suppose.

C'est sur ce fond de guerre d'images médiatiques et de rivalités politiques qu'Henri Gaden et Henri Gouraud vont prendre une grande série de photographies documentant le trajet de Samori Touré prisonnier.

\section{Le portrait d'un vaincu}

Signalons d'emblée que les photographies de Samori Touré sont très nombreuses, depuis sa capture en septembre 1898 jusqu'à son départ en exil à partir de Saint-Louis, en passant par son jugement à Kayes devant le général de Trentinian ${ }^{8}$. Elles connaîtront un vif succès et seront diffusées ensuite sur

7. «Prince des croyants ».

8. Peu d'images sur l'Almami circulent auparavant. Notons toutefois, sur gravure, le « portrait de Bissandougou » (pour une analyse de ce portrait, voir RicHEMOND [2014]). Il y a une controverse sur l'attribution de certaines photographies représentant Samori Touré. Bien que de nombreuses photographies de Samori Touré soient présentes dans les fonds 
cartes postales dans la collection Fortier ${ }^{9}$, la plus riche collection concernant l'Afrique, ainsi que par des reproductions en gravures dans des journaux, des mémoires d'officiers, ou encore des illustrés. Devenues anonymes ${ }^{10}$, elles forment une vaste « imagerie coloniale » dont la paternité revient en réalité pour une large part à Henri Gaden, ainsi qu'à Henri Gouraud. Ces photographies ont été tellement reprises, copiées, gravées, rééditées qu'elles ont perdu leur légende et leur attribution, ce qui était commun à l'époque.

Tout au long du trajet de Samori Touré, de septembre à novembre 1898, Gaden et Gouraud ont pris des centaines de clichés. Ceux-ci ont documenté la vie de la Colonne, le passage des cours d'eau, le repos des troupes, la vie des villages, mais aussi et surtout le quotidien de leur prisonnier illustre. De nombreux fonds d'archives ont conservé leurs négatifs, ce qui rend la tâche de reconstitution parcellaire et complexe. Gouraud avait des doubles des clichés de Gaden dans son immense collection de clichés que Julie d'Andurain a éditée (d'Andurain 2016: 59-64 sur Samori Touré). Ce sont les archives municipales de Bordeaux qui conservent le fonds Gaden, avec 335 clichés stéréoscopiques qui ont servi de matière pour une exposition réalisée par Alain Ricard (Ricard \& Jégo 2001) en 2011 au Salon du livre de Bordeaux ${ }^{11}$, parmi lesquels figurent les clichés représentant Samori. Des positifs et des négatifs de cette même série se trouvent également dans les Archives nationales d'Outre-mer (ANOM), dans la section des archives privées de Gaden ${ }^{12}$. En outre, elles sont également reproduites dans un album photo ayant appartenu à de Lartigue, conservé au Service historique de la Défense (SHD), dont la cote indique que les photographies ont été prises par Gouraud ${ }^{13}$. Il y a donc un conflit d'attribution de ces photographies, dont certaines sont devenues très célèbres, ce qui fait singulièrement écho aux rivalités entre les officiers concernant l'attribution de la capture de Samori. Le lieutenant Rampont,

privés de Gaden, à Aix-en-Provence et à Bordeaux, il ne semble pas que ce soit lui qui ait pris les plus célèbres clichés de son prisonnier. Du 29 septembre 1898 au 17 octobre, Samori Touré, Gaden et Gouraud font route commune jusqu'à Beyla, et le 26 octobre Samori Touré part pour Kayes, mais Gaden reste sur place. Les portraits les plus connus auraient été pris ultérieurement sur le trajet, vraisemblablement à Saint-Louis, entre le 4 janvier 1899, date de son arrivée, et le 5 février, date de son embarquement à Dakar pour le Gabon. Je remercie Odile Goerg pour les informations sur cette controverse.

9. " Samory à Saint-Louis », " Arrivée de Samory à Saint-Louis », "Samory écoutant la sentence le condamnant à l'exil à Kayes (Haut-Sénégal), déc. 1898 » (Collection Gilles Le Ouzon).

10. Richemond (2014 : 22-28) reprend les légendes des cartes postales.

11. Clichés numérotés 2718-3385, sans classement.

12. ANOM 15APC/2. Papiers Gaden : photographies de Samori non datées, non localisées.

13. SHD Vincennes, GR2K194 album photo «Prise de Samory ». 166 photos, 1898, prises par Gouraud. Album ayant appartenu à de Lartigue. 
dans le même fonds du SHD, dispose de vingt-deux clichés de Samori Touré bien moins connus, qui ont été peu diffusés ${ }^{14}$. Enfin, retrouvées par hasard lors de nos recherches, six photographies du capitaine Tamburini figuraient dans un Coran ayant appartenu à Samori Touré, dans le fonds du Musée de 1'Armée, aux Invalides ${ }^{15}$. Émouvantes, ces six photographies documentent l'attente de Samori Touré à Saint-Louis du Sénégal avant son départ en exil, dans les jours précédant ou suivant immédiatement sa tentative de suicide ${ }^{16}$. C'est un homme défait et résigné qui fixe l'objectif, sa « favorite » en retrait, debout au fond de la pièce.

Ainsi donc, tous les officiers français ayant côtoyé Samori Touré en ont fait un sujet photographique. Cela indique, d'une part, à quel point les officiers étaient friands de ce passe-temps au moment de la conquête coloniale ${ }^{17}$ et, d'autre part, qu'ils avaient conscience de documenter leur propre parcours et de contrôler la construction de leur image médiatique. Photographier à l'époque coloniale un vaincu aussi célèbre que Samori Touré, c'est en ce sens magnifier celui qui est derrière l'objectif : celui qui a le pouvoir de capturer l'instant de la défaite de son ennemi. C'est attester de son propre pouvoir.

Une photographie parmi toutes celles qui ont été prises a eu un destin iconique particulier : le fameux portrait au Coran de Samori Touré. Immédiatement diffusée en carte postale (fig. 2), elle orne notamment les premières de couverture des récits consacrés à la conquête (Gouraud 1939). Dans la masse des clichés pris par tous les officiers français, pourquoi est-ce celle-ci qui est passée à la postérité ? Pourquoi celle-ci précisément plutôt que d'autres portraits pris au cours du même trajet?

Sur l'image, Samori Touré est assis. Contrairement à de nombreux autres portraits d'anonymes pris par Henri Gouraud, qui sont souvent de trois-quarts, Samori fixe ici l'objectif frontalement. Détendu, il esquisse un sourire à celui qui le prend en photo, et qui a dû s'accroupir pour être à sa hauteur. Il porte un turban sombre comme à son habitude (Person 1968, II : 842-843), opérant un contraste avec la cotonnade de couleur claire de sa longue robe, par ailleurs rayée de très fines bandes noires. Il tient entre ses genoux un Coran, bien

14. SHD Vincennes, GR2K292. Photographies du lieutenant Rampont. 22 photographies du Soudan et de Samory.

15. Invalides, Musée de l'Armée. Inv. 12375-1/7.

16. Cette tentative de suicide a également fait l'objet d'une illustration dans la presse, avide de sensationnalisme : Le Petit Journal, Supplément illustré, 5 février 1899, p. 48, « Tentative de suicide de Samory ", pleine page couleur.

17. Sur l'album photographique de la Mission Marchand en 1899, voir Deroo (2010) qui le publie dans une riche édition illustrée. Sur les voyageurs et la photographie, voir le catalogue d'exposition, «Des voyageurs à l'épreuve du terrain : études, enquêtes, explorations. 1800-1960» (Archives nationales 2016). 
visible, dont il semble avoir interrompu la lecture pour fixer le spectateur, et qu'il laisse légèrement choir, de sorte que la page en caractères arabes occupe le premier plan. D'autres portraits de l'empereur le montrent assis, parmi ses fils ou ses proches, au repos ou en marche. Le portrait reproduit dans Lectures pour tous. Revue Universelle illustrée en regard du journal de marche de Gouraud (1899b) le montre sur une chaise, avec un chapelet, une jambe encore posée à terre, regardant sur le côté. Dans le portrait au Coran, au contraire, Samori Touré pose ostensiblement. Il fixe celui qui le regarde. Il décide d'offrir à l'objectif une image de chef digne, lettré, instruit, serein, encore souverain de sa personne. Puisqu'il n'est en réalité plus souverain que de lui-même, ayant désormais perdu son empire, il profite de sa position de sujet photographique pour prendre possession de son image ${ }^{18}$. C'est certainement cela qui frappe dans ce cliché : la maîtrise que le sujet (dans tous les sens du terme) prend de sa pose. En quelque sorte, Samori Touré s'appropriant le medium photographique, renverse momentanément le rapport de pouvoir et convertit les officiers français en instrument de sa propre image. Subalterne, indéniablement - son statut de prisonnier de guerre ne change évidemment pas avec ce cliché - , le sujet fait trembler un instant le rapport hiérarchique qui l'unit au photographe ${ }^{19}$.

\section{La fabrique d'une icône à l'époque de la reproductibilité technique: une politique de la répétition}

\section{Dupliquer en série la résistance anticoloniale}

Les autres photographies des séries prises par Henri Gouraud ou par d'autres officiers français présentent Samori Touré comme un vaincu. Dans ces mises en scènes, sa capture est l'incarnation de la victoire française sur le territoire, sa photographie illustre la conquête coloniale. Les autres clichés le mettent en scène entre des tirailleurs sur le trajet vers Kayes, ou bien apprenant sa condamnation à l'exil face à Trentinian, où il est définitivement défait et

18. Comparable en cela à ce que B. HIRSCH (1995:37-49) note à propos de Ménélik, même si celui-ci exerçait encore une puissance réelle au moment des prises de vue : «Il est certain par contre que Ménélik est le souverain éthiopien qui a compris le parti que l'on pouvait tirer de l'image photographique, celui qui a accepté de se montrer à des fins de propagande et qui laissait volontiers les voyageurs étrangers photographier les attributs de sa puissance.»

19. R. VoKes (2013: 9) note que, dans certaines enquêtes photographiques dans la période coloniale en Centrafrique, "African subjects did exert some degree of agency over the photographic process. [...] Africans did sometimes actively shape the encounter. » 
vaincu, ou encore entouré d'une foule de curieux débarquant à Saint-Louis, enfin entre ses geôliers au Gabon. Toutes ces photographies ont en commun d'avoir connu une diffusion importante sur carte postale pendant la période coloniale. Toutes affirment avec force la suprématie coloniale française sur les territoires nouvellement conquis. Le portrait au Coran est singulier en cela qu'il laisse possible une autre interprétation, postcoloniale, au sens où elle prend le contrepied de sa lecture coloniale.

C'est cette image que choisira Sékou Touré pour inverser les significations que lui avaient attribuées les officiers français : le portrait ne sera plus l'exemple type de l' " ennemi de la France » réduit en captivité, comme l'indique la légende de la carte postale, mais bien l'illustration du résistant à la colonisation dont la lutte héroïque constitue un exemple à suivre pour la nation. Il y a un retournement radical du sens de l'image après l'indépendance de la Guinée, acquise en 1958, avec le NON au référendum (Goerg, Pauthier \& Diallo 2010). Le vaincu d'hier devient l'ancêtre fondateur d'aujourd'hui (Bertho 2016). Samori Touré, par cette photographie, devient le porteur de la contestation anticoloniale. Sékou Touré a fait de ce cliché une icône qui a la particularité de contester sa signification initiale.

Par sa « reproductibilité technique» (Benjamin [1939] $2000: 274-275$ ), l'image perd son lien avec le « hic et nunc de 1'original ». En dupliquant à l'envi cette photographie célèbre, Sékou Touré établit en réalité un double geste : d'une part, il inverse le sens du cliché (vaincu/vainqueur), d'autre part, il fournit une « aura $»^{20}$ à la figure qu'il contribue à créer - celle de son ancêtre dans la résistance anticoloniale. C'est précisément dans et par la répétition que l'icône se construit : elle en tire sa force. «En permettant à la reproduction de s'offrir au récepteur dans la situation où il se trouve, elle actualise 1'objet reproduit » (ibid. : 276). Or, il est intéressant de voir que cette actualisation de l'objet s'établit ici en lien avec une inversion de sa signification. La photographie reste la même, mais sa duplication dans un autre contexte suffit à en renverser les modalités d'interprétation, sans avoir besoin d'opérer à des retouches ou à de quelconques modifications.

Dans les manuels scolaires de l'indépendance (Niane \& Suret-Canale 1961), dans les publications d'État ${ }^{21}$, sur les pochettes des vinyles, sur des timbres, le

20. Tandis que Walter Benjamin précisait que, dans la sérialité de la reproduction, « ce qui dépérit dans l'œuvre d'art, c'est son aura » ([1939] $2000: 276$ ), il nous semble, au contraire, que c'est l'hypothèse inverse qui rend compte de l'usage contestataire de la photographie de Samori Touré par Sékou Touré : c'est la répétition et la sérialité qui confèrent au portrait son aura puisqu'il construit son personnage en même temps qu'un récit national qui se légitime dans sa propre répétition.

21. Voir l'ouvrage sans date, sans auteur, Regard sur le passé. L'Almamy Samory Touré 18301900, Service national des Arts et de la Culture (division des arts plastiques et expositions), 
portrait au Coran est partout, avec des cadrages plus ou moins rapprochés sur le visage. Devenu une icône du régime socialiste, le portrait sert également de modèle à la statuaire qui semble n'avoir eu, concernant Samori Touré, qu'un seul modèle tant la répétition à l'identique est flagrante: si l'on compare le buste du Musée National à Kaloum au buste du mausolée dans l'enceinte de la Grande Mosquée Fayçal, ou encore le buste de la place de Kankan, l'on constate qu'il s'agit dans les trois cas d'une recréation en trois dimensions tout à fait identique au portrait au Coran. Le rond-point du 2 octobre, dans le quartier de Moussoudougou à Conakry, est orné depuis 1998 pendant le « Centenaire du Souvenir » (de sa capture en 1898), d'une statue en pied de Samori Touré, mais la pose, le buste, les traits du visage, les couleurs correspondent à la photographie originale. Cette reprise et ces variations autour du même thème illustrent à quel point le modèle iconographique établi par Sékou Touré dispose encore à la fin des années 1990 d'une aura suffisamment importante pour que les commanditaires de la statue ne s'en éloignent pas.

Seule la statue équestre de Samori Touré s'éloigne de ce canon établi par le président: entièrement dorée, cette statue signale l'entrée du camp militaire Almamy Samory Touré, ce qui justifie très certainement la présence du cheval (fig. 3, 4, 5).

\section{Enjeux politiques du montage postcolonial}

Pour Sékou Touré, disposer d'un tel fonds documentaire sur Samori Touré a été une grande chance. La photographie atteste : elle est pour lui une preuve de la réalité de la résistance anticoloniale. En quelque sorte, elle constitue un témoignage du récit national que le régime socialiste élabore - même si ce récit se fait au prix d'une réinterprétation complète des clichés coloniaux. Lue en ce sens, l'image a un double rôle: elle « documente un moment de 1'histoire » (Didi-Huberman 2009: 33-34), mais alliée à un montage, « elle induit éventuellement une réflexion plus poussée ». Ce que fait Sékou Touré de ces images coloniales, c'est précisément une opération de «montage » pour leur donner une portée anticoloniale : la simple transposition sur les documents, vinyles, manuels scolaires, timbres (fig. 6, 7) produits par le régime socialiste suffisent à en inverser la signification. La multiplication de ce montage se fait par un usage politique de la sérialité. Cette lecture contestataire du cliché colonial se légitime dans et par la sérialité : c'est la répétition qui finit par attester de la lecture socialiste de cette photographie

ministère de la Jeunesse, des Arts et des Sports, Conakry. Album photographique avec une préface d'Ahmed Sékou Touré. 
qui devient ainsi une icône nationale voire panafricaine (Goerg 2011 : 84 ; Pauthier 2014). Or, il faut également prendre garde à faire l'histoire de ce montage (ou à montrer le montage), qui est, lui aussi, situé historiquement dans un contexte donné : c'est une histoire de lectures contestataires des images qu'il s'agit alors d'écrire.

\title{
Mémoires d'un portrait, mémoires d'un régime: circulations postcoloniales des images coloniales
}

\author{
Mémoire d'une famille: les Touré, entre nostalgie et ambition \\ pédagogique
}

Icône du régime socialiste de Sékou Touré, le portrait de Samori Touré a tellement contribué à l'instauration d'un « panthéon national » que l'un et l'autre sont liés dans la mémoire collective. En particulier, la famille de l'ancien dirigeant reste extrêmement attachée à la figure de Samori Touré et singulièrement au portrait au Coran. Mohamed Touré, secrétaire général du PDG-RDA (Parti démocratique de Guinée-Rassemblement démocratique africain), atteste de l'importance de la figure de Samori Touré et de la très bonne réception qu'a eue l'œuvre de l'historien Yves Person (1968-1975) auprès des ministres et de 1'entourage proche de Sékou Touré ${ }^{22}$. Mohamed Lamine Touré, petit-fils de Sékou Touré, dernier gouverneur de la Banque centrale pendant le régime socialiste, se souvient également d'avoir été mandaté par son grand-père pour écrire une lettre de remerciements à Yves Person ${ }^{23}$. La famille Touré rédige depuis 2000 un journal mensuel afin de diffuser les nouvelles concernant les mariages, les baptêmes, les promotions et les avancements de carrière de ses membres. La première page du numéro 251 du mois de décembre 2016 (fig. 8) reprend cette même photographie ${ }^{24}$. Après les nouvelles des cotisations de l'association familiale et diverses informations privées, deux articles « de fond » sont mis en regard : l'un sur Napoléon, l'autre sur Samori Touré — ce qui justifie la couverture - qui est une large citation de plusieurs pages de Samori, une révolution dyula d'Yves Person (1968-1975). Les deux empereurs ont souvent été comparés l'un à l'autre, le second étant qualifié régulièrement de « Napoléon des savanes » (Kourouma 2003 : 140-151). Pour la famille

22. Entretien avec Mohamed Touré réalisé avec Marie Rodet à Conakry au siège du PDGRDA, le 22 décembre 2016.

23. Entretien avec Mohamed Lamine Touré réalisé avec Marie Rodet à son domicile à Conakry, le 23 décembre 2016.

24. Nous remercions la famille Touré de nous avoir gracieusement fourni le dernier numéro de leur journal. 
Touré, la photographie de Samori Touré constitue bel et bien toujours une icône, symbolisant l'appartenance à une lignée prestigieuse dont la puissance est attestée par le souvenir des grandes figures historiques. L'historiographie qui leur est attachée (Yves Person et la réception de son œuvre) appartient également au patrimoine mémoriel de la famille: Mohamed Lamine Touré possède les trois tomes de Samori, une révolution dyula, qu'il se souvient avoir achetés à l'IFAN à Dakar en 1980. En cela, le portrait au Coran est un marqueur identitaire pleinement investi par les membres de la famille, qui contribuent activement à la transmission du souvenir de leur aïeul.

Autre aspect de cette «volonté pédagogique » citée par les descendants, la constitution d'un musée dont la vocation est d'être ouvert aux enseignants et à leurs classes (fig. 9). Donnant sur la rue, au pied du domicile de Mohamed Lamine Touré, deux vastes salles mettent à la disposition du public plusieurs ouvrages dont les œuvres de Sékou Touré, les romans de grands intellectuels africains des années 1970 et 1980, et certains classiques socialistes. Au mur sont reproduits les portraits de résistants à la colonisation, dont ceux pris par Henri Gaden et Henri Gouraud. Samori Touré y occupe une large place, et d'autres photographies coloniales le représentant sont également reproduites, comme celle de son exil au Gabon. À ses côtés notamment, Lat Dior, dont le portrait est une copie de gravure. Le parallèle entre les deux figures de résistants est à cet égard intéressant : il n'existe pas de photographie coloniale de Lat Dior, c'est donc toujours la même gravure qui est reproduite. L'imagerie en est singulièrement plus pauvre que pour Samori Touré.

La famille Touré explique à quel point ce musée et leur journal répondent à une ambition pédagogique de diffusion du savoir historiographique sur l'histoire africaine et à une volonté de réhabilitation de héros panafricains (ou perçus comme tels $)^{25}$. Ils se plaignent en effet de campagnes de diffamation à l'encontre de ces héros : Mohamed Lamine Touré raconte, par exemple, à quel point il souffrait, étant écolier, d'être connu comme le descendant de Samori Touré que ses camarades et le maître d'école accusaient d'être un tyran. Le musée joue dès lors un rôle de réappropriation de l'histoire africaine, à différentes échelles, familiale, nationale, panafricaine. Le fait que ce musée familial soit souvent désert est le sujet de plaintes de la famille, qui déplore que les jeunes générations ne s'intéressent pas à l'histoire africaine, ce qui va de pair avec une profonde nostalgie du régime de Sékou Touré, où ces héros étaient davantage mis en avant dans la sphère médiatique.

25. Entretien avec Mohamed Lamine Touré (op. cit.). 


\section{Circulations sur Internet: reprises et répétitions}

C'est bien sûr sur Internet que les circulations sont les plus nombreuses. Le portrait y est répété régulièrement, servant à illustrer de nombreux sites d'encyclopédies participatives ${ }^{26}$ ou de vulgarisation de l'histoire africaine ${ }^{27}$. En plan rapproché sur son visage ou bien encore colorisée ${ }^{28}$, les réappropriations du portrait sont nombreuses et se légitiment souvent par leur entre-citation. L'extrême régularité de l'iconographie surprend : c'est très majoritairement le portrait au Coran qui est dupliqué, alors qu'il existe d'autres photographies du personnage. C'est certainement que l'image a acquis un tel statut d'icône qu'elle devient presque un attendu de la consommation de biens culturels sur Internet concernant Samori Touré.

Sur Facebook, le portrait est inséré dans des montages d'autres photographies coloniales de lui dans des diaporamas à sa gloire : la page « Guinée Conakry » suivie par environ 250000 personnes ${ }^{29}$ poste régulièrement des contenus relatifs à Samori Touré. Le 9 novembre 2016, le 29 janvier 2017, le 5 février 2017, le site a publié des montages photographiques illustrant le parcours du « résistant le plus redoutable que les Français eurent à affronter en Afrique de l'Ouest », chacun de ces posts étant «likés » entre 1000 et 1700 fois, ce qui donne une ampleur du phénomène de réutilisation de la mémoire historique sur les réseaux sociaux. Les dizaines de commentaires sont très

26. Sur Wikipedia, <https://fr.wikipedia.org/wiki/Samory_Tour\%C3\%A9>, consulté le 3 juin 2017. Légende du portrait de Samori : «Samory, le Coran entre les mains. »

27. Nous pourrions multiplier les exemples, tant les sites sont nombreux et similaires. Pour donner un aperçu de cette production médiatique, nous opérons ici une sélection de cas représentatifs. Blog « Hérodote.net», <https://www.herodote.net/29_septembre_1898-evenement-18980929.php>, article « 29 septembre 1898, capture de Samory Touré », consulté le 3 juin 2017. La photographie est tronquée pour ne présenter que le visage de l'empereur, en plan rapproché. Ce même plan rapproché se retrouve également sur ce site de vulgarisation $<\mathrm{http}$ //afrikhepri.org/samory-toure-1830-1900/>, consulté le 3 juin 2017. Le site militant $<\mathrm{http}$ ://www.une-autre-histoire.org/samory-toure-biographie/>, consulté le 3 juin 2017, reprend aussi ce plan rapproché. Notons que le sous-titre du blog est le suivant : «Une autre histoire, histoires oubliées, histoires occultées » ce qui indique la posture postcoloniale du blog, au sens où il tend à vulgariser d'une contre-écriture de l'histoire. Cette posture critique passe sous silence les usages faits de la figure de Samori Touré sous Sékou Touré.

28. Le site « Sénégalmetis », <http://senegalmetis.com/C1_Samory_Toure.html $>$, consulté le 3 juin 2017, offre une version colorisée du portrait réalisé par Henri Gaden, où le turban de l'Almamy est rouge, ce qui semble être un contresens historique puisque Yves Person soulignait que Samori Touré affectionnait les turbans noirs. Le coloriste a certainement tenté de donner un dynamisme visuel par ces coloris fantaisistes.

29. <https://www.facebook.com/LaGuinee224/?hc_ref=SEARCH>, consulté le 3 juin 2017. 
régulièrement élogieux — ce qui ne serait bien sûr pas le cas s'il s'agissait d'une page malienne ${ }^{30}$.

D'autres usages prennent des libertés avec le portrait. Le site Classic Afrobeat ${ }^{31}$ présente, par exemple, un spectacle musical inspiré du célèbre «Regard sur le passé » du Bembeya Jazz National («Regard sur le passé, musique épique en trois mouvements », par Sekouba Bambino et Baba Sissoko, dirigé par Marco Zanotti). Le portrait sert d'affiche au spectacle, dont les contours sont colorisés en vert et ocre, avec une esthétique inspirée de l'art nouveau assez réussie.

Le calme du portrait au Coran «chiffre » donc, pour reprendre les termes de Jacques Rancière, l'histoire d'une rencontre entre des hommes qui se sont appréciés : un prisonnier de guerre et ses gardiens. Marquée par une théâtralisation extrêmement importante, les rapports hiérarchiques entre ces hommes ne seront pas modifiés par ce cliché, bien évidemment. Simplement, par l'attention qu'il porte à la mise en scène de soi dans cette pose choisie avec soin, Samori Touré trouve un moyen de suspendre temporairement la sentence de son exil : il sourit au spectateur.

Ce sourire serein, énigmatique, aura un destin iconique remarquable et les circulations postcoloniales de ce cliché illustrent un parcours médiatique important. Caractéristique d'une réappropriation postcoloniale ${ }^{32}$, ces «montages » de significations superposées interrogent également la mémoire de l'histoire coloniale, voire de l'historiographie, avec le grand rôle d'Yves Person notamment.

Ainsi, l'image contestataire n'est pas toujours celle que l'on croit : bras levé lançant un pavé dans une manifestation immortalisé par Gilles Caron, cette contestation peut aussi être exprimée par un sourire, geste plus discret s'il en est, mais qui porte en lui un pouvoir tout aussi important de réappropriation de la mise en scène de son propre corps. Théâtraliser cet instant fugace, c'est, pour Samori Touré, le moyen d'échapper temporairement au statut de prisonnier, tandis qu'il est confronté à une sentence d'exil qu'il récusera toute sa vie. Cet instant de la pose, au calme impressionnant, sera chargé de significations

30. Sur la mémoire de Samori Touré au Mali, voir Rodet \& Challier (2014).

31. <https://classicafrobeat.com/regard-sur-le-passe/regard-sur-le-passe-francais/>, consulté le 3 juin 2017.

32. Ce cas n'est pas propre à la Guinée. De la même manière, C. GoRE (2001) illustre comment les clichés de l'Oba Ovonramwen du Bénin furent réappropriés dans le Nigéria postcolonial, en s'insérant dans des stratégies de commémorations importantes. L'ampleur de ces usages est néanmoins certainement d'une moindre ampleur que sous Sékou Touré. 
diverses au cours du siècle, dans des usages contestataires variés : anticoloniaux, anti-impériaux, nationalistes, panafricains. Ces photographies n'ont pas cessé d'être réutilisées, et leurs significations d'être remodelées à chaque fois. La contestation n'est donc pas portée par le geste en soi mais bien par l'intention qui guide le cliché, et ensuite, par chacun de ses usages.

Les Afriques dans le monde (LAM), CNRS, Bordeaux.

Fig. 1. - Une, Le Petit Journal, Supplément illustré, 30 octobre 1898, « CAPTURe De SAmory Par Le Lieutenant JACQUin »

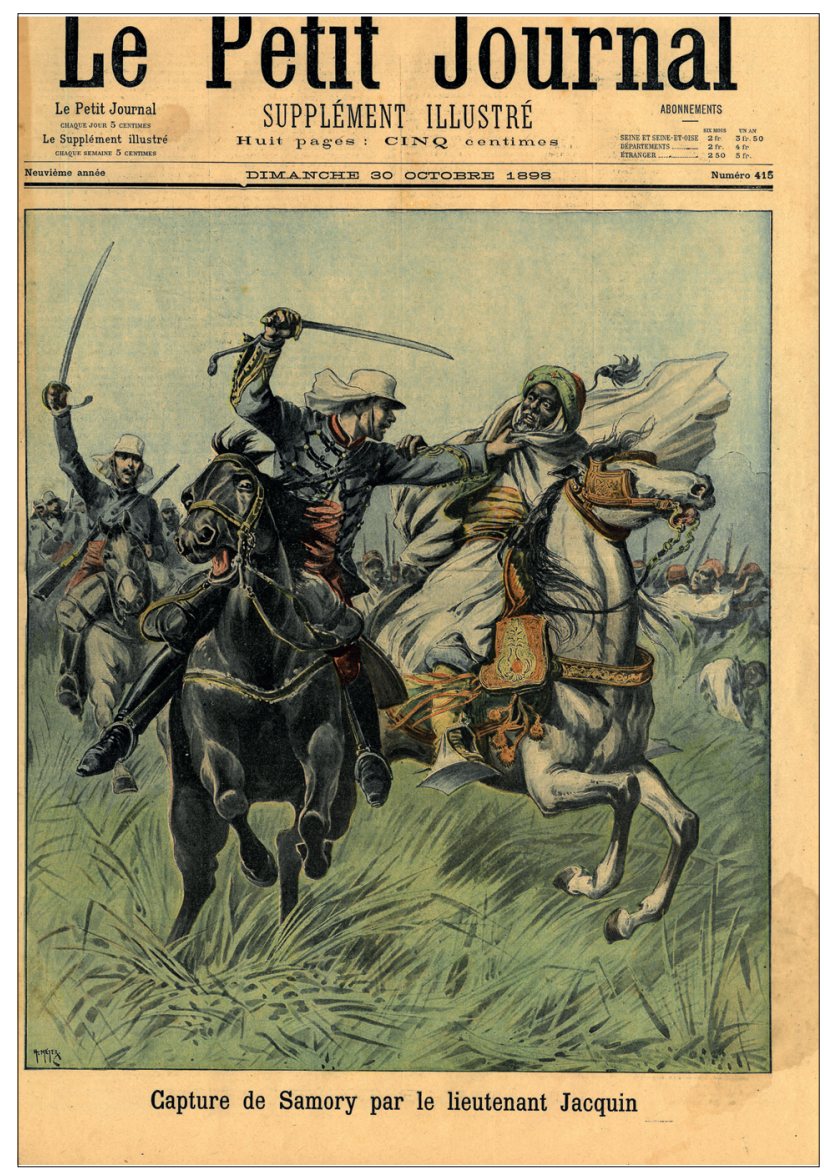

Collection personnelle d'E. Bertho. 
Fig. 2. — « 1278. Afrique Occidentale — SAmory — Dioula Soudanais DeVenu un puissant Almamy, ENNEMi DE LA France. CAPTURÉ EN 1898 »

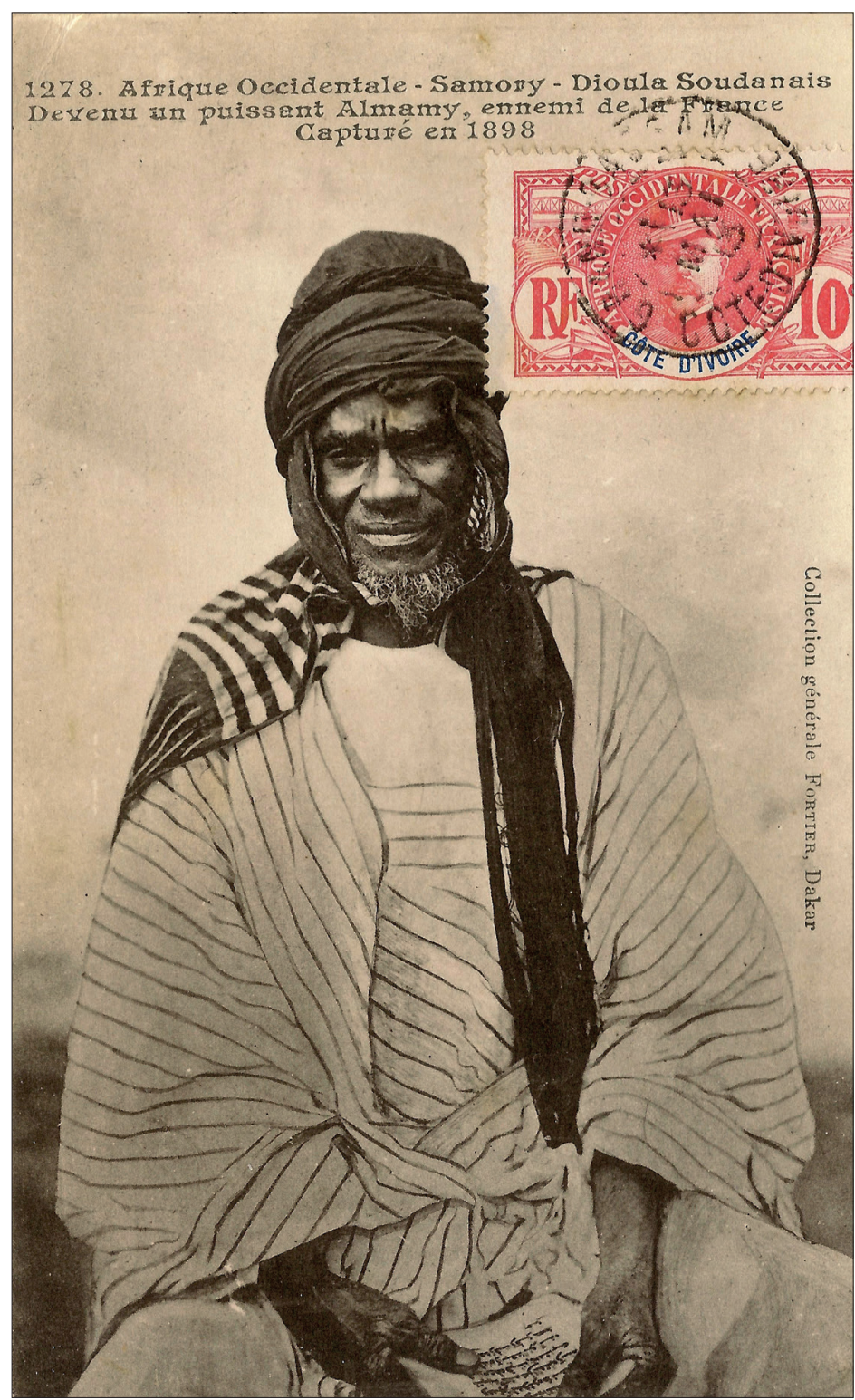

Collection générale Fortier, Dakar (collection personnelle d'E. Bertho). 


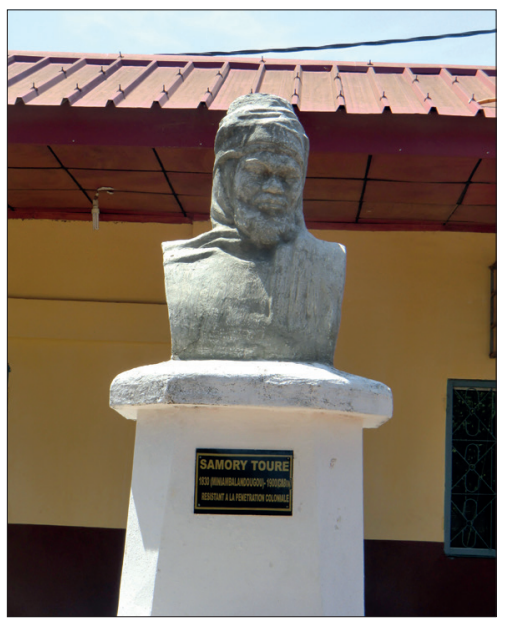

Fig. 3. - Buste de SAmori Touré, Musée National, Conakry

Fig. 4. - Buste de SAmori Touré, Mausolée de SÉKou Touré, SAMORI
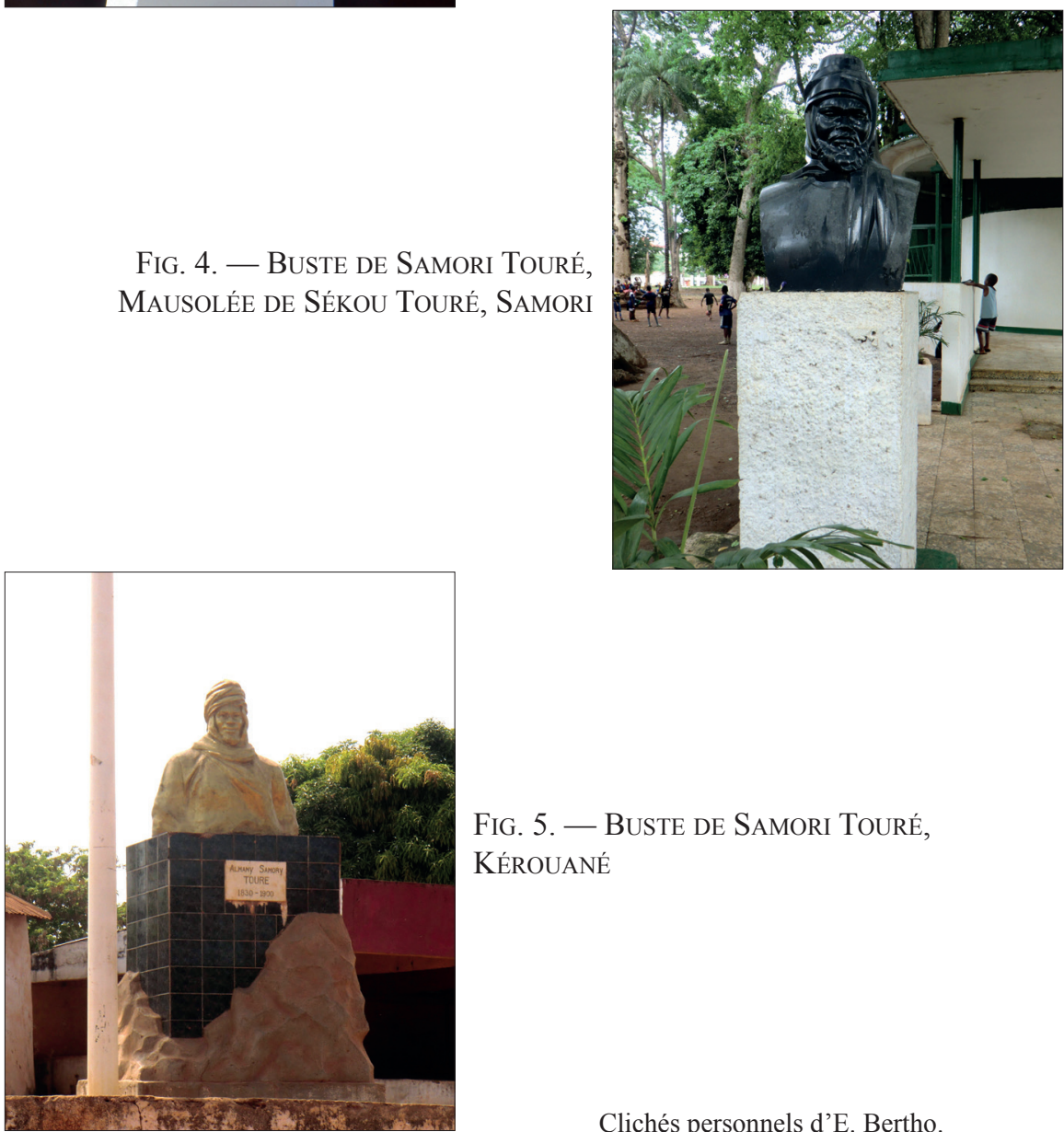

Fig. 5. - Buste de SAmori Touré, KÉROUANÉ 
Fig. 6. - Timbre de SAMORI Touré, 1962, GuinÉE.

SÉrIE « HÉros ET MARTYR DE L’AfriQue » (YT : GN : 118)

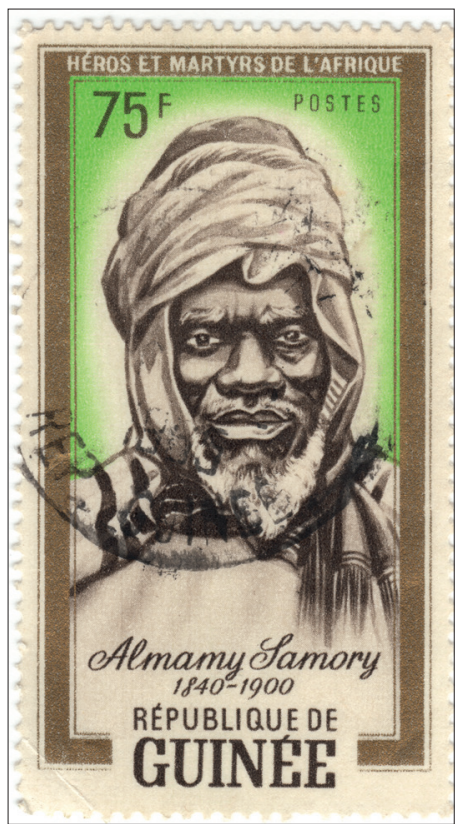

Fig. 7. - Timbre de SAmori Touré, 1978, BÉnin.

SÉRIE « Héros Africain de LA RÉSISTANCE À L'INVASION COLONIALE » (YT 412)

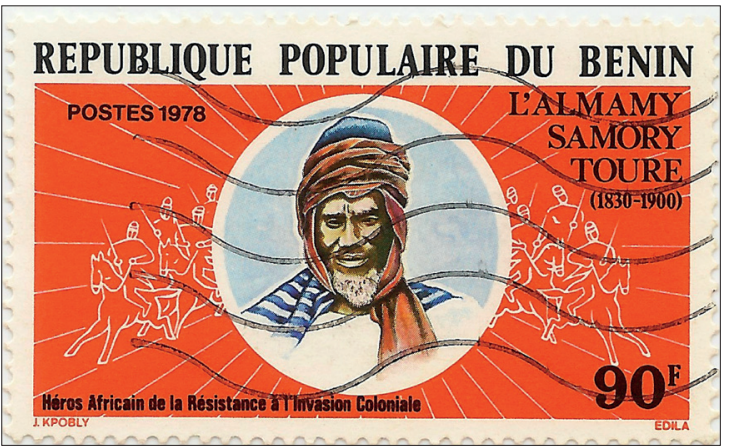

Collection personnelle d'E. Bertho. 
Fig. 8. - Frontispice du Journal Mensuel de LA Famille Touré, $\mathrm{N}^{\circ} 251$ DU 4 DÉCEMBRE 2014

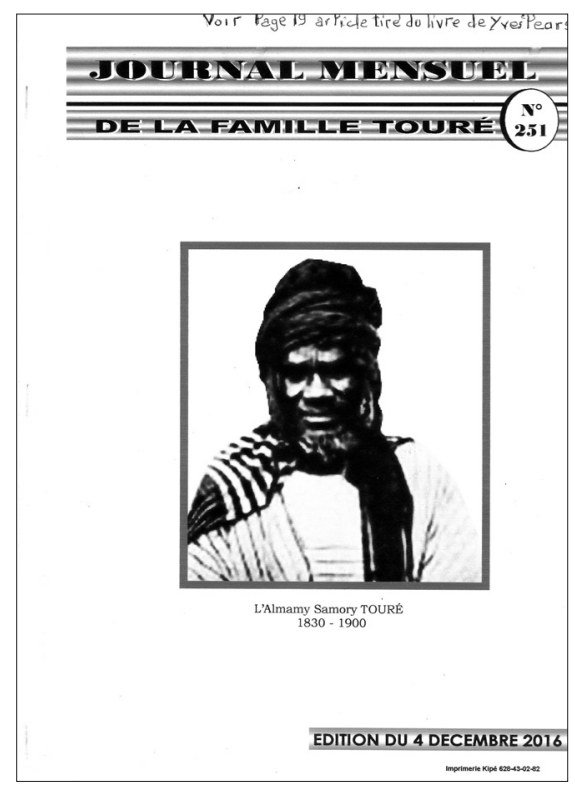

Collection personnelle d'E. Bertho.

Fig. 9. - Musée De La famille Touré À LA GLOIRE DES RÉSisTANTS À LA PÉNÉTRATION COLONIALE. CONAKRY, DÉCEMBRE 2016

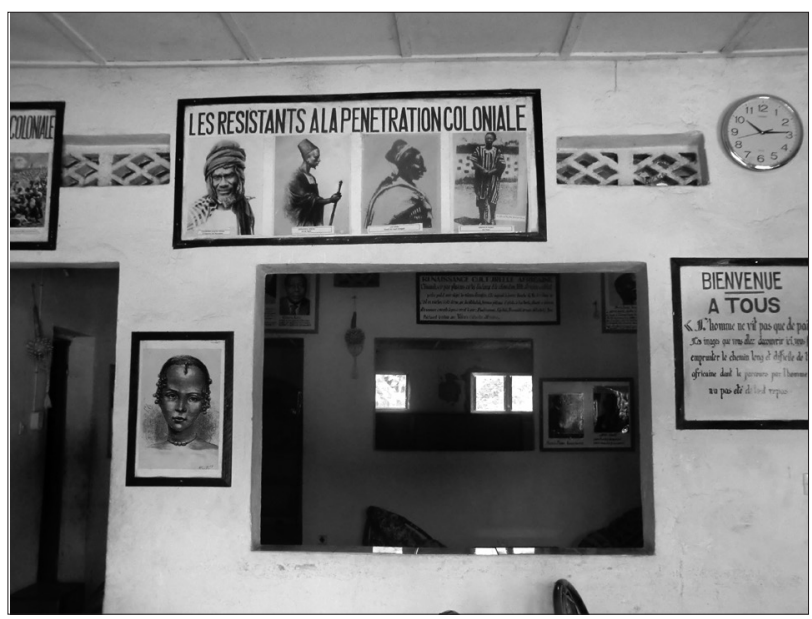

Cliché personnel d'E. Bertho. 


\section{BIBLIOGRAPHIE}

D’ANDuRAIn J., 2012, La capture de Samory, 1898: L'achèvement de la conquête de l'Afrique de l'Ouest, Saint-Cloud, Soteca.

- 2016, Henri Gouraud. Photographies d'Afrique et d'Orient. Trésors des archives du Quai d'Orsay, Paris, Pierre de Taillac.

Archives Nationales, 2016, Des voyageurs à l'épreuve du terrain : études, enquêtes, explorations, 1800-1960, Pierrefitte-sur-Seine, Éditions Archives Nationales.

BANCEL N., Blanchard P. \& GeRVEREAU L. (DIR.), 1993, Images et colonies : iconographie et propagande coloniale sur l'Afrique française, 1880-1962, Paris, BDIC-ACHAC.

BARthes, R., 1980, La chambre claire. Note sur la photographie, Paris, Gallimard.

BENJAMIN W., [1939] 2000, «L'œuvre d'art à l'époque de sa reproductibilité technique », in Euvres III, Paris, Folio : 269-316.

— 2000 [1940], « Sur le concept d'histoire», in Euvres III, Paris, Folio : 427-443.

Bertho E., 2015, « Médias, Propagande, Nationalismes. La filiation symbolique dans les chants de propagande : Robert Mugabe et Mbuya Nehanda, Ahmed Sékou Touré et Samory Touré », numéro spécial «Paroles publiques, paroles confidentielles », Cahiers de littérature orale, 77-78 : 171-193.

— 2016, «Mémoires postcoloniales et figures de résistants africains dans la littérature et dans les arts. Nehanda, Samori, Sarraounia comme héros culturels », Thèse de doctorat, Paris, Paris 3-Sorbonne Nouvelle.

Deroo, E., 2010, La grande traversée de l'Afrique, mission Marchand: 1896-1899, Congo, Fachoda, Dibouti, Paris, Little Big Man.

Didi-Huberman G., 2009, Quand les images prennent position, L'œil de l'histoire, 1, Paris, Éditions de Minuit.

Didi-Huberman G. ET AL. (DIR.), 2016, Soulèvements, Paris, Gallimard-Jeu de Paume.

Dilley R., 2015. Henri Gaden à travers l'Afrique de l'Ouest (1894-1939) : fils de Bordeaux, aventurier africain, Paris, L'Harmattan.

Goerg O., Pauthier C. \& Diallo A., 2010, Le non de la Guinée (1958), Entre mythe, relecture historique et résonances contemporaines, Paris, L'Harmattan.

Goerg O., 2011, «Couper la Guinée en quatre : ou comment la colonisation a imaginé l'Afrique », Vingtième siècle. Revue d'histoire critique, 111 : 73-88.

Gore C., 2001, «Commemoration, Memory and Ownership: Some Social Contexts of Contemporary Photography in Benin City, Nigeria », Visual Anthropology, 14 (3) : 321-342. 
Gouraud H., 1899a, «Journal de marche», Bulletin du Comité de l'Afrique française, $1: 7-13$.

— 1899b, « Journal de marche », Lectures pour tous. Revue Universelle illustrée : 961-976.

— 1939, Au Soudan: souvenirs d'un Africain, Paris, P. Tisné.

HiRSCH B., 1995, «Les photographies du gebbi de Ménélik à Addis Ababa: Une première approche », in A. Triulzi (ed.), Fotografia e Storia dell'Africa, Naples, Atti del Convegno Internazionale Napoli-Roma 1992 : 37-49.

Kourouma A., 2003, «Les derniers jours de Samory. Le Capitaine Gouraud, vainqueur du "Napoléon des savanes" $1898 »$, in P. FourNiÉ (dir.), Aventuriers du monde : Les grands explorateurs français au temps des premiers photographes : 1866-1914, Paris, L'Iconoclaste : 140-151.

Niane D. T. \& Suret-Canale J., 1961, Histoire de l'Afrique Occidentale, Paris, Présence africaine.

PAUTHIER C., 2012. «Forger l'imaginaire national : Les enjeux de l'enseignement en histoire en République de Guinée au lendemain de l'indépendance », in C. LABRUNEBadiane, M. A. De SuREmain \& P. Bianchini (dir.), L'école en situation postcoloniale, Paris, L'Harmattan : 57-80.

— 2014, L'indépendance ambigue. Construction nationale, anticolonialisme et pluralisme culturel en Guinée (1945-2010), Thèse de doctorat, Paris, Université Paris Diderot.

Person Y., 1968-1975, Samori : une révolution dyula, 3 t., Mémoires de l'Institut fondamental d'Afrique noire, Dakar, IFAN ; Paris, Centre de recherches africaines.

RANCIÈRE J., 2013, Le destin des images, Paris, La Fabrique éditions.

RicARD A. \& JÉGO A.-L., 2001, Henri Gaden photographe [exposition au Salon du livre de Bordeaux 2001], Bordeaux, Éditions Confluences.

Richemond S., 2012, "Samory Touré - Le Portrait de Bissandougou », Images et Mémoires, $32: 28-30$.

— 2014, «Iconographie de Samory Touré : De Guélémou à Kayes (1898)», Images et Mémoires, $41: 22-28$.

Rodet M. \& Challier F., 2014, The Diambourou. Slavery and Emancipation in Kayes - Mali, London, SOAS.

Touré A. S., 1969, La révolution culturelle. t. XVII, Cuvres, Conakry, Imprimerie nationale Patrice Lumumba.

— 1972, Poèmes militants, Conakry, Parti démocratique de Guinée, Imprimerie nationale Patrice Lumumba.

Tupitsyn M., 2006, "Against the Camera: For the Photographic Archive », in C. Merewether (ed.), The Archive, Documents of Contemporary Art, Cambridge, MIT Press : 103-107. 
Venayre S., 2016, Une guerre au loin: Annam, 1883, Paris, Les Belles Lettres.

VOKEs R., 2013, Photography in Africa : Ethnographic Perspectives, Woodbridge, James Currey.

RÉSUMÉ

À partir d'un important travail d'archives, nous proposons de retracer les parcours et usages des photographies de Samori Touré lors de sa capture, en croisant les différents acteurs qui I'ont accompagné: Henri Gaden, Henri Gouraud, et le capitaine Tamburini entre autres. Le fameux portrait au Coran, parmi ces différentes séries de clichés de 1898 et 1899, a connu un vif succès populaire dès l'époque coloniale, via des cartes postales, des reproductions en illustrés ou dans des magazines. Ce cliché se retrouve également dans des mémoires ou souvenirs d'officiers. À l'indépendance, Sékou Touré, qui se revendique d'une filiation symbolique de Samori Touré, fait un usage extrêmement important de ce portrait, tout en inversant la lecture: ce n'est plus une prise de guerre mais la célébration d'un père de la nouvelle patrie, mort en martyr et en exil en 1900, dont il s'agit de magnifier la mémoire (par la musique ou encore la statuaire).

Mots-clés: Guinée, Samori Touré, Sékou Touré, circulation, photographie, postcolonial.

\section{ABSTRACT}

Photographs of Samori Touré: From the Colonial Postcard to Record Covers. Circulations of an Iconic Picture. - Based on significant archival research, we analyze the circulations and uses of colonial photographs of Samori Touré shortly after his capture. We retrace the various officers who escorted him and those who took pictures of him: Henri Gaden, Henri Gouraud, Captain Tamburini, among others. In particular, the famous portrait with the Koran was a tremendous success during the colonial period and was frequently reproduced on postcards or in illustrated magazines. This picture was also reproduced in memoirs of French officers. After the independence, Sékou Touré who claimed that Samori was his ancestor, widely used the image. The meaning of Samori's portrait in this postcolonial context was inverted: no longer a victim or a captive of the colonial French army, but a founding father of the newborn nation who died a martyr in exile in 1900. The picture was used to magnify his memory, on record covers or in statues. 